\begin{tabular}{|c|l|}
\hline Title & $\begin{array}{l}\text { Mouse al dehyde dehydrogenase A L DH3B2 is local ized to lipid droplets via two C-terminal try ptophan residues and } \\
\text { lipid modification }\end{array}$ \\
\hline Author(s) & Kitamura, Takuya; Takagi, Shuyu; Naganuma, Tatsuro; Kihara, A kio \\
\hline Citation & $\begin{array}{l}\text { Biochemical journal, 465, 79-87 } \\
\text { https:/doi.org/10.1042/BJ20140624 }\end{array}$ \\
\hline Issue Date & 2015-01-02 \\
\hline Doc URL & http://hdl.handle.net/2115/60410 \\
\hline Rights & The Version of Record (VoR) is avail lable at www.biochemj.org \\
\hline Type & article (author version) \\
\hline File Information & manuscript.pdf \\
\hline
\end{tabular}

Instructions for use 


\section{Mouse aldehyde dehydrogenase ALDH3B2 is localized to lipid droplets via two C-terminal tryptophan residues and lipid modification}

\section{Takuya Kitamura*, Shuyu Takagi ${ }^{*}$, Tatsuro Naganuma*, and Akio Kihara*1}

* Laboratory of Biochemistry, Faculty of Pharmaceutical Sciences, Hokkaido University, Kita 12-jo, Nishi 6-chome, Kita-ku, Sapporo 060-0812, Japan

${ }^{1}$ To whom correspondence should be addressed:

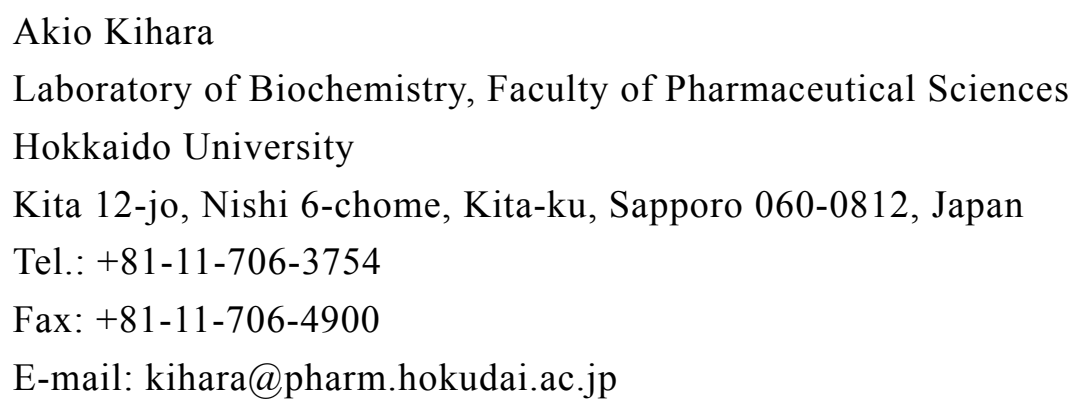

Short title: Lipid droplet localization of ALDH3B2

Summary statement: The mouse aldehyde dehydrogenases ALDH3B2 and ALDH3B3 exhibit similar substrate specificity but distinct intracellular localization (ALDH3B2, lipid droplets; ALDH3B3, plasma membrane). The C-terminal prenylation and two Trp residues are important for the lipid droplet localization of ALDH3B2. 


\section{ABSTRACT}

Aldehyde dehydrogenases (ALDHs) catalyze the conversion of toxic aldehydes to non-toxic carboxylic acids. Of the 21 ALDHs in mice, it is the ALDH3 family members (ALDH3A1, ALDH3A2, ALDH3B1, ALDH3B2, and ALDH3B3) that are responsible for the removal of lipid-derived aldehydes. However, ALDH3B2 and ALDH3B3 have yet to be characterized. Here, we examined the enzyme activity, tissue distribution, and subcellular localization of ALDH3B2 and ALDH3B3. Both were found to exhibit broad substrate preferences from medium-chain to long-chain aldehydes, resembling ALDH3A2 and ALDH3B1. Although ALDH3B2 and ALDH3B3 share extremely high sequence similarity, their localizations differed, with ALDH3B2 found in lipid droplets and ALDH3B3 localized to the plasma membrane. Both ALDH3B2 and ALDH3B3 were modified by prenylation at their C-termini; this modification greatly influenced their membrane localization and enzymatic activity toward hexadecanal. We found that their C-terminal regions, particularly the two Trp residues (Trp462 and Trp469) of ALDH3B2 and the two Arg residues (Arg462 and Arg463) of ALDH3B3, were important for the determination of their specific localization. Abnormal quantity and perhaps quality of lipid droplets are implicated in several metabolic diseases. We speculate that ALDH3B2 acts to remove lipid-derived aldehydes in lipid droplets generated via oxidative stress as a quality control mechanism.

Keywords: aldehyde dehydrogenase; lipid; lipid droplet; lipid modification; prenylation; protein targeting

The abbreviations used are: ABE, acyl-biotinyl exchange; ALDH, aldehyde dehydrogenase; EGFP, enhanced green fluorescent protein; ER, endoplasmic reticulum; 4-HNE, 4-hydroxy-2-nonenal; S1P, sphingosine-1-phosphate. 


\section{INTRODUCTION}

The many aldehyde molecules present in living organisms are either absorbed from external sources such as diet, drugs, and environmental pollutants or endogenously produced via metabolism of lipids, amino acids, and neurotransmitters or through oxidation of biomolecules [1]. Aldehyde molecules are reactive in nature. The carbonyl carbon of aldehydes is partially positive-charged and is readily attacked by nucleophiles such as amines, producing a Schiff base. Therefore, aldehydes can easily react with important cellular biomolecules and impair their functions. Aldehyde dehydrogenases (ALDHs) are responsible for the removal of aldehydes: they oxidize the toxic aldehydes to form non-toxic carboxylic acid. There are 19 ALDH and 21 Aldh genes in the human and mouse genomes, respectively. The cytotoxicity of aldehyde molecules is evidenced by the fact that mutations in human $A L D H$ genes can give rise to several inherited disorders, such as Sjögren-Larsson syndrome (ALDH3A2) [2], type II hyperprolinemia (ALDH4A1) [3], pyridoxine-dependent seizures (ALDH7A1) [4], $\gamma$-hydroxybutyric aciduria (ALDH5A1) [5], and methylmalonic aciduria (ALDH6A1) [6].

The ALDH3 subfamily in particular plays important roles in the oxidation of lipid-derived aldehydes. Five ALDH3 subfamily members exist in mice: namely, ALDH3A1, ALDH3A2, ALDH3B1, ALDH3B2, and ALDH3B3, with ALDH3B3 being rodent-specific. ALDH3A1/crystallin is a soluble protein abundantly expressed in the cornea [7], and protects the cornea and lens from UV-induced oxidative stress by removing oxidative products such as lipid-derived 4-hydroxy-2-nonenal (4-HNE) [8]. Accordingly, Aldh3a1-knockout mice develop lens opacification and cataracts in response to UV exposure [8]. ALDH3A2 is expressed ubiquitously and exhibits activity toward saturated and unsaturated aliphatic aldehydes of $\mathrm{C} 6$ to $\mathrm{C} 24$ in length [9]. The major splicing isoform of ALDH3A2 is a type II endoplasmic reticulum (ER) membrane protein, whereas its minor isoform is localized to peroxisomes [10, 11]. A variety of ALDH3A2 substrates are generated through the metabolism of lipids such as fatty alcohols, leukotriene $\mathrm{B}_{4}$, phytol, and sphingosine-1-phosphate (S1P) and through lipid peroxidation [12-14].

ALDH3B1 is also expressed ubiquitously, and its protein levels are high in the kidney, liver, and ovary $[15,16]$. Similar to ALDH3A2, human ALDH3B1 exhibits activity toward medium-chain to long-chain aldehydes; in contrast to ALDH3A2, its subcellular localization is in the plasma membrane [17]. It follows that there is a clear distinction between the roles of ALDH3B1 and ALDH3A2: they act to detoxify aldehydes generated in the plasma membrane and the ER (and peroxisomes), respectively. Indeed, ALDH3B1 protects cells from the cytotoxicity of exogenously added aldehydes and oxidants [16]. We recently reported that human ALDH3B1 is dually lipidated (palmitoylation and prenylation) and that prenylation of ALDH3B1 is important for its plasma membrane localization and its activity toward long-chain aldehydes [17].

Lipid droplets store abundant triglycerides for membrane synthesis and as an energy supply, and imbalances of lipid droplets and fat storage are associated with several metabolic diseases [18, 19]. The structure of lipid droplets is unique in that they consist of a hydrophobic core of neutral lipids surrounded by a monolayer of phospholipids, mainly 
phosphatidylcholine [20]. Lipid droplets are ubiquitous and are particularly prominent in adipose tissue. The most abundant fatty acid in adipose tissues (i.e., the most abundant triglyceride fatty acid in lipid droplets) is oleic acid, comprising $\sim 44 \%$ of total adipose tissue fatty acids [21]. Although much progress has been made in understanding lipid droplet biogenesis within the last decade, many unknown factors still exist, including the mechanism of protein targeting to lipid droplets and the quality control of lipids in lipid droplets. Considering the abundance of unsaturated fatty acids in lipid droplets, oxidative stress may produce fatty aldehydes from peroxidized lipids. However, the ALDHs responsible for the removal of such aldehydes in lipid droplets are not known.

ALDH3B2 and ALDH3B3 are expected to exhibit activity toward lipid-derived aldehydes due to their high sequence similarities to other ALDH3 family members. However, these two ALDHs have yet to be characterized. Therefore, analysis of both members is crucial to understanding the overall cellular defensive mechanism against aldehydes in greater detail.

In the present study, we characterized ALDH3B2 and ALDH3B3 with respect to their activity, subcellular localization, tissue distribution, and lipid modification. We found that both ALDH3B2 and ALDH3B3 exhibited broad substrate specificity toward medium-chain and long-chain aldehydes, similar to ALDH3A2 and ALDH3B1. ALDH3B2 was localized to lipid droplets, whereas ALDH3B3 was localized to the plasma membrane. These results indicate that ALDH3 subfamily members function to remove lipid-derived aldehydes in specific organelles: i.e., ALDH3A1 in the cytosol, ALDH3A2 in the ER and peroxisome, ALDH3B1 and ALDH3B3 in the plasma membrane, and ALDH3B2 in lipid droplets. 


\section{MATERIALS AND METHODS}

\section{Cell culture and transfection}

HEK $293 \mathrm{~T}$ and HeLa cells were cultured in DMEM (Sigma, St. Louis, MO) supplemented with $10 \% \mathrm{FBS}, 100 \mathrm{U} / \mathrm{ml}$ penicillin, and $100 \mu \mathrm{g} / \mathrm{ml}$ streptomycin. HEK 293T cells were grown in dishes pre-coated with $0.3 \%$ collagen. Transfections were conducted using Lipofectamine Plus Reagent (Life Technologies, Carlsbad, CA), according to the manufacturer's instructions.

\section{Plasmids}

The pCE-puro 3xFLAG-1 [22] and pEGFP-C1 (Clontech, TAKARA Bio, Palo Alto, CA) plasmids are mammalian expression vectors designed to produce proteins tagged with an $\mathrm{N}$-terminal 3xFLAG and an N-terminal enhanced green fluorescent protein (EGFP), respectively. Mouse Aldh3a1 (GenBank accession number, AF072815), Aldh3a2 (AF289813), Aldh3b1 (BC046597), Aldh3b2 (AK136505), and Aldh3b3 (AK081788) genes were amplified by PCR using the respective forward (-F1) and reverse (-R1) primers listed in Table 1 from mouse tissue cDNAs (for Aldh3a1, skeletal muscle; for Aldh3a2, liver; for Aldh3b1 and Aldh3b3, brain; and for Aldh3b2, lung) (Clontech, TAKARA Bio). Human CGI-58/ABHD5 gene was amplified by PCR using CGI-58-F1 and CGI-58-R1 primers (Table 1) from human placenta cDNA (Clontech, TAKARA Bio). The amplified fragments were first cloned into the pGEM-T Easy vector (Promega, Madison, WI), generating the pGEM-mALDH3A1, pGEM-mALDH3A2, pGEM-mALDH3B1, pGEM-mALDH3B2, pGEM-mALDH3B3, and pGEM-CGI-58 plasmids. The cDNA fragment was then prepared from each resulting plasmid by digesting with the appropriate restriction enzymes and cloning into either pCE-puro $3 \times$ FLAG-1 or pEGFP-C1, generating the pCE-puro 3xFLAG-mALDH3A1, pCE-puro 3xFLAG-mALDH3A2, pCE-puro 3xFLAG-mALDH3B1, pCE-puro 3xFLAG-mALDH3B2, pCE-puro 3xFLAG-mALDH3B3, pCE-puro 3xFLAG-CGI-58, pEGFP-mALDH3B1, pEGFP-mALDH3B2, and pEGFP-mALDH3B3 plasmids.

The ALDH3B2/ALDH3B3 chimeras were constructed by exchanging the StuI-SpeI, StuI-HindIII, or HindIII-BamHI fragment of either the pGEM-mALDH3B2 or pGEM-mALDH3B3 plasmid for the corresponding fragment of the other gene. The chimera genes generated were digested with BamHI and SalI and cloned into the BglII-SalI site of the pEGFP-C1 plasmid, generating the pEGFP-mALDH3B(3/2/2), pEGFP-mALDH3B(2/3/2), pEGFP-mALDH3B(2/2/3), pEGFP-mALDH3B(2/3/3), pEGFP-mALDH3B(3/2/3), and pEGFP-mALDH3B(3/3/2) plasmids.

Most of the Aldh3b2 and Aldh3b3 mutants were created using a QuikChange site-directed mutagenesis kit (Stratagene, Agilent Technologies, La Jolla, CA) with the appropriate template plasmids and primers: for Aldh3b2 W462A, primers ALDH3B2-Q1F and ALDH3B2-Q1R; for Aldh3b2 W469A and Aldh3b2 W462/469A, primers ALDH3B2-Q2F and ALDH3B2-Q2R; and for Aldh3b3 R462/463A, primers ALDH3B3-Q1F and ALDH3B3-Q1R (Table 1). The Aldh3b2 C476S mutant was generated by PCR using the primers ALDH3B2-F1 and ALDH3B2-R2 from the pGEM-mALDH3B2 plasmid. 


\section{In vitro ALDH assay}

3xFLAG-ALDH3A1，3xFLAG-ALDH3A2，3xFLAG-ALDH3B1，3xFLAG-ALDH3B2, and 3xFLAG-ALDH3B3 were expressed in HEK 293T cells. Cells were suspended in buffer A (50 $\mathrm{mM}$ HEPES-NaOH ( $\mathrm{pH}$ 7.4), $150 \mathrm{mM} \mathrm{NaCl}, 10 \%$ glycerol, 1x protease inhibitor mixture (Complete EDTA-free; Roche Diagnostics, Indianapolis, IN), $1 \mathrm{mM}$ PMSF, and $1 \mathrm{mM}$ DTT) containing $1 \%$ Triton X-100 and sonicated. After incubation for $30 \mathrm{~min}$ at $4{ }^{\circ} \mathrm{C}$, the total cell lysates were centrifuged at $100,000 \mathrm{~g}$ for $30 \mathrm{~min}$ at $4{ }^{\circ} \mathrm{C}$. The supernatant was then incubated with anti-FLAG M2 affinity agarose gel (Sigma) overnight at $4{ }^{\circ} \mathrm{C}$ with rotation. Beads were washed three times with buffer A containing $0.1 \%$ Triton $\mathrm{X}-100$, and bound proteins were eluted with buffer A containing $100 \mu \mathrm{g} / \mathrm{ml} 3$ xFLAG peptide and $0.1 \%$ Triton X-100.

The in vitro ALDH assay was performed by incubating $10 \mathrm{ng}$ of the affinity-purified protein with $500 \mu \mathrm{M} \mathrm{NAD}^{+}$and $100 \mu \mathrm{M}$ hexadecanal [23], octanal (Wako Pure Chemical Industries, Osaka, Japan), or acetaldehyde (Wako Pure Chemical Industries) in buffer B (50 $\mathrm{mM}$ Tris- $\mathrm{HCl}$ (pH 8.5), $150 \mathrm{mM} \mathrm{NaCl}, 10 \%$ glycerol, and $0.1 \%$ Triton X-100) for $15 \mathrm{~min}$ at $37{ }^{\circ} \mathrm{C}$. The reaction was monitored by measuring the fluorescence of the NADH product (excitation at $356 \mathrm{~nm}$ and emission at $460 \mathrm{~nm}$ ) using a monochromator Infinite M200 (Tecan, Männedorf, Switzerland).

\section{Cell fractionation}

Cells treated with or without the geranylgeranyltransferase I inhibitor GGTI-2133 (Sigma) were suspended in buffer A and lysed by sonication. After removal of unlysed cells by centrifugation at $1,000 \mathrm{~g}$ for $3 \mathrm{~min}$ at $4{ }^{\circ} \mathrm{C}$, the resulting supernatant (total lysate) was centrifuged at $100,000 \mathrm{~g}$ for $30 \mathrm{~min}$ at $4{ }^{\circ} \mathrm{C}$. The resulting pellet (i.e., the membrane fraction) and supernatant (i.e., the soluble fraction) were subjected to immunoblotting.

\section{Immunoblotting}

Proteins were separated by SDS-PAGE and electrotransferred to an Immobilon PVDF membrane (Millipore, Billerica, MA) at a constant current of $2 \mathrm{~mA}$ per $1 \mathrm{~cm}^{2}$ membrane for 1 $\mathrm{h}$. The resulting membrane was incubated with a 5\% skim milk blocking solution (dissolved in TBS-T (20 mM Tris- $\mathrm{HCl}(\mathrm{pH} 7.5), 137 \mathrm{mM} \mathrm{NaCl}$, and $0.05 \%$ Tween 20) for $1 \mathrm{~h}$ at room temperature. The membrane was then incubated with blocking solution containing anti-FLAG M2 $(1 \mu \mathrm{g} / \mathrm{ml}$; Stratagene, Agilent Technologies), anti-calnexin (H-10; $0.2 \mu \mathrm{g} / \mathrm{ml}$; Santa Cruz Biotechnology, Santa Cruz, CA), or anti-GAPDH (1 $\mu \mathrm{g} / \mathrm{ml}$; Ambion, Austin, TX) antibodies for $1 \mathrm{~h}$ at room temperature. After washing with TBS-T three times, the membrane was incubated with blocking solution containing HRP-conjugated anti-rabbit or anti-mouse IgG $\mathrm{F}(\mathrm{ab})_{2}$ fragment (each 1:7500 dilution; GE Healthcare Life Sciences, Buckinghamshire, UK) antibodies for $1 \mathrm{~h}$ at room temperature, followed by washing with TBS-T three times. Labeling was detected using Pierce ECL Western Blotting Substrate (Thermo Fisher Scientific, Waltham, MA).

\section{Fluorescence microscopy}

HeLa cells were grown on coverslips and transfected with the indicated plasmids. Cells were 
fixed with $3.7 \%$ formaldehyde in PBS for $10 \mathrm{~min}$ at $37{ }^{\circ} \mathrm{C}$ and permeabilized with $0.1 \%$ Triton X-100 in PBS for $5 \mathrm{~min}$ at room temperature. After washing with $60 \%$ isopropanol, cells were incubated with $1.8 \mathrm{mg} / \mathrm{ml}$ oil red $O$ (Nacalai Tesque, Kyoto, Japan) in $60 \%$ isopropanol for $20 \mathrm{~min}$ at room temperature, followed by repeat washing with PBS. Cells were then blocked with blocking solution $\left(10 \mathrm{mg} / \mathrm{ml} \mathrm{BSA}\right.$ in PBS) at $37{ }^{\circ} \mathrm{C}$ for $30 \mathrm{~min}$ and incubated with anti-FLAG M2 antibody $(2 \mu \mathrm{g} / \mathrm{ml})$ in blocking solution for $1 \mathrm{~h}$ at room temperature. After washing three times with PBS, the cells were incubated with Alexa Fluor 488- or 594-conjugated anti-mouse antibody (each at $5 \mu \mathrm{g} / \mathrm{ml}$; Molecular Probes, Life Technologies, Eugene, OR) in blocking solution for $1 \mathrm{~h}$ at room temperature. Coverslips were washed with PBS, mounted with Prolong Gold Antifade Reagent (Molecular Probes, Life Technologies) and observed under a Leica DM5000B microscope (Leica Microsystems, Wetzlar, Germany). In the case of EGFP-fusion protein-expressing cells, the permeabilization, blocking, and antibody staining steps were omitted.

\section{Real-time quantitative RT-PCR}

Total RNA was isolated from tissues of 4-8 week-old C57BL/6 mice using a NucleoSpin RNA II kit (MACHEREY-NAGEL, Dueren, Germany), according to the manufacturer's instructions. Real-time quantitative PCR was performed using the one-step SYBR PrimeScript RT-PCR kit II (TaKaRa Bio, Shiga, Japan) and respective forward (-rt1) and reverse (-rt2) primers (Table 1) on a CFX96TM Real-Time PCR detection system (Bio-Rad Laboratories, Hercules, CA), according to the manufacturer's instructions. The transcript level of each gene was normalized with that of GAPDH. The RT reaction was performed at $42{ }^{\circ} \mathrm{C}$ for $5 \mathrm{~min}$, followed by incubation at $95{ }^{\circ} \mathrm{C}$ for $10 \mathrm{~s}$, and then the PCR reaction consisting of 40 cycles of incubation at $95{ }^{\circ} \mathrm{C}$ for $5 \mathrm{~s}, 64{ }^{\circ} \mathrm{C}$ for $30 \mathrm{~s}$, and $72{ }^{\circ} \mathrm{C}$ for $30 \mathrm{~s}$.

\section{Acyl-biotinyl exchange (ABE) assay}

The assay was performed as described previously [24]. 


\section{RESULTS}

ALDH3B2 and ALDH3B3 exhibit enzymatic activity toward medium- and long-chain aldehydes

To examine the activity of ALDH3B2 and ALDH3B3, N-terminally 3xFLAG-tagged ALDH3B2 and ALDH3B3, as well as ALDH3A1, ALDH3A2, and ALDH3B1 for comparison, were affinity-purified and subjected to in vitro enzyme assays using a short-chain aldehyde (acetaldehyde), a medium-chain aldehyde (octanal), and a long-chain aldehyde (hexadecanal) as substrates. Acetaldehyde was not good substrate for the ALDH3 family members in general, with only ALDH3B1 exhibiting a weak activity toward acetaldehyde (Figure 1A). ALDH3B1, ALDH3B2, and ALDH3B3 exhibited similar activity profiles, with greater activity toward hexadecanal than octanal. ALDH3A2 exhibited comparable but still slightly higher activity toward hexadecanal than octanal. On the other hand, the activity of ALDH3A1 was much higher toward octanal than hexadecanal, reflecting its localization in the cytosol. These substrate preferences of ALDH3A1, ALDH3A2, and ALDH3B1 were consistent with previous studies $[9,17,25]$. These results indicate that ALDH3A2, ALDH3B1, ALDH3B2, and ALDH3B3 have activity toward medium- and long-chain aldehydes in common.

We next examined the expression of mouse ALDH3B2 and ALDH3B3 mRNAs by real-time quantitative RT-PCR in the liver, kidney, lung, testis, small intestine, white adipose tissue, and spleen (Figure 1B). The highest expression of ALDH3B2 was observed in the testis, followed by the white adipose tissue, while its expression in the liver was low. The expression of $A L D H 3 B 3$ mRNA was the highest in the testis, followed by the kidney, and low in the liver and lung.

\section{ALDH3B2 is localized to lipid droplets}

Distinct subcellular localizations have been reported for ALDH3A1 (cytosol) [26], ALDH3A2 (ER and peroxisomes) [10,11], and human ALDH3B1 (plasma membrane) [17]. However, the localization of ALDH3B2 or ALDH3B3 had yet to be identified. To determine the intracellular localization of these two enzymes, we first fractionated the total cell lysate prepared from HeLa cells expressing 3xFLAG-ALDH3 proteins into soluble and membrane fractions. Consistent with current knowledge of localization, cytosolic ALDH3A1 was detected in the soluble fraction and the integral membrane protein ALDH3A2 was recovered in the membrane fraction (Figure 2A). Mouse ALDH3B1 was localized to both the soluble and membrane fractions (Figure 2A), as was human ALDH3B1, which anchored to the plasma membrane via lipidation [17]. Both ALDH3B2 and ALDH3B3 exhibited fractionation patterns similar to ALDH3B1.

We next examined the intracellular localization of the N-terminally EGFP-tagged ALDH3B1, ALDH3B2, and ALDH3B3 in HeLa cells using fluorescence microscopy (Figure 2B). Although EGFP alone was localized to the cytosol and nucleus, EGFP-ALDH3B1 and EGFP-ALDH3B3 were found in the plasma membrane as well as in some punctate structures in the cytoplasm. EGFP-ALDH3B2 formed ring-like structures, reminiscent of lipid droplets. To confirm the observation that ALDH3B2 localized to lipid droplets, HeLa cells expressing $3 x F L A G-A L D H 3 B 2$ were treated with oleic acids to promote the development of lipid 
droplets, and then incubated with oil red $O$ to stain them. Indirect immunofluorescence microscopy revealed that ALDH3B2-positive ring structures surrounded the oil red $O$-stained lipid droplets (Figure 2C). Furthermore, ALDH3B2 was co-localized with the lipid droplet marker CGI-58 (Figure 2D). These results indicated that ALDH3B2 is localized to lipid droplets.

\section{ALDH3B proteins are geranylgeranylated}

We have previously shown that human ALDH3B1 is dually lipidated (via palmitoylation and prenylation) [17]. Prenylation (farnesylation or geranylgeranylation) occurs at C-terminal CaaX motifs ( $\mathrm{C}$, prenylated Cys residue; a, an aliphatic amino acid residue), with the $\mathrm{X}$ residue determining the type of prenylation, i.e., geranylgeranylation ( $\mathrm{X}=\mathrm{Leu}$ or Ile) or farnesylation (X = Ser, Met, Gln, or Cys) [27]. Mouse ALDH3B1, ALDH3B2, and ALDH3B3 all contain C-terminal CTLL sequences, as does human ALDH3B1. Therefore, these ALDH3B proteins are also predicted to be modified by geranylgeranylation. To confirm this supposition, cells were treated with the geranylgeranyltransferase I inhibitor GGTI-2133 and subjected to a cell fractionation assay. Upon treatment with GGTI-2133, membrane-associated ALDH3B1, ALDH3B2, and ALDH3B3 decreased compared with mock-treated cells, while their respective soluble forms increased (Figure 3A). Thus, ALDH3B1, ALDH3B2, and ALDH3B3 are indeed geranylgeranylated.

We next examined the effects of the loss of geranylgeranylation on the enzymatic activity of mouse ALDH3B proteins. Treatment with GGTI-2133 reduced the activity of ALDH3B1, ALDH3B2, and ALDH3B3 toward hexadecanal (Figure 3B). This effect has been similarly observed in human ALDH3B1 [17]. These results indicate that geranylgeranylation is important for the membrane localization and enzyme activity of ALDH3B proteins.

Cys residues adjacent to a prenylated Cys residue are often palmitoylated [28], as is the case with human ALDH3B1 [17]. Human ALDH3B1 contains one Cys residue (Cys463), which is located two amino acids before the N-terminal of the prenylated Cys residue (Cys465), whereas mouse ALDH3B1 contains two Cys residues (Cys462 and Cys463) near the CTLL prenylation sequence. On the other hand, no Cys residues exist adjacent to the CTLL sequences in ALDH3B2 or ALDH3B3. An acyl-biotinyl exchange (ABE) assay, which substitutes biotin for the palmitate moiety [29], revealed that ALDH3B1 was palmitoylated, but not ALDH3B2 or ALDH3B3 (Figure 3C). We created Ser-substituted mutants for the putative palmitoylation sites of ALDH3B1 (C462S and C463S single substitutions and a C462/463S double substitution) and subjected them to the ABE assay. Palmitoylation decreased in the single mutants (C462S and C463S) and was completely abolished in the double mutant (C462/463S) (Figure 3D), indicating that ALDH3B1 is palmitoylated at the Cys462 and Cys463 residues.

\section{The C-terminal regions of ALDH3B2 and ALDH3B3 determine their localization}

Although ALDH3B2 and ALDH3B3 share extremely high sequence similarity (86.4\% identity and $92.5 \%$ similarity), their intracellular localizations differ, with ALDH3B2 found in lipid droplets and ALDH3B3 localized to the plasma membrane (Figure 2). To identify the region 
that is necessary for ALDH3B2 to localize to the lipid droplets, we created several chimera proteins of ALDH3B2 and ALDH3B3 by exchanging the $\mathrm{N}$-terminal region (amino acid residues 1-26), the middle region (27-452), and the C-terminal region (453-479) with each other. The constructed chimera proteins were termed $\operatorname{ALDH} 3 \mathrm{~B}(3 / 2 / 2), \operatorname{ALDH} 3 \mathrm{~B}(2 / 3 / 2)$, $\operatorname{ALDH3B}(2 / 2 / 3), \operatorname{ALDH} 3 \mathrm{~B}(2 / 3 / 3), \operatorname{ALDH} 3 \mathrm{~B}(3 / 2 / 3)$, and $\operatorname{ALDH} 3 \mathrm{~B}(3 / 3 / 2)$ : for example, $\operatorname{ALDH} 3 \mathrm{~B}(2 / 3 / 2)$ means that the chimera protein contained the $\mathrm{N}$-terminal region of $\mathrm{ALDH} 3 \mathrm{~B} \underline{2}$, the middle region of $\mathrm{ALDH} 3 \mathrm{~B} \underline{\underline{3}}$, and the $\mathrm{C}$-terminal region of ALDH3B$\underline{2}$. Exchanging the $\mathrm{N}$-terminal regions did not affect the localization of ALDH3B2 or ALDH3B3, since $\operatorname{ALDH} 3 \mathrm{~B}(3 / 2 / 2)$ and $\operatorname{ALDH} 3 \mathrm{~B}(2 / 3 / 3)$ exhibited intracellular localization that was similar to the original ALDH3B2 and ALDH3B3 proteins, respectively (Figure 4). Substitution of the middle region also had little effect on the localization of ALDH3B2 or ALDH3B3. ALDH3B(2/3/2) exhibited lipid droplet localization similar to ALDH3B2. $\operatorname{ALDH} 3 \mathrm{~B}(3 / 2 / 3)$ was localized in the plasma membrane, although some lipid droplet localization was also observed (Figure 4).

In contrast, exchanging the $\mathrm{C}$-terminal regions greatly influenced their localization. $\operatorname{ALDH} 3 \mathrm{~B}(3 / 3 / 2)$ was localized to lipid droplets, similar to ALDH3B2. ALDH3B(2/2/3) was mainly localized to the plasma membrane, but also partly to lipid droplets (Figure 4). These results suggest that the most important region determining lipid droplet localization exists in the C-terminal region of ALDH3B2, although the middle region appears to be partially involved in the localization as well.

The C-terminus of ALDH3B3 contains three Arg residues, whereas ALDH3B2 only has one (Figure 5A). It has been reported that clusters of positively charged residues near prenylation sites are important for the plasma membrane localization of K-RasB and Cdc42 $[30,31]$. Therefore, to examine the role of Arg residues in the plasma membrane localization of ALDH3B3, we created a double Ala-substituted mutant (R462/463A), in which both the Arg462 and Arg463 residues were substituted with Ala residues. The plasma membrane localization of ALDH3B3 was diminished in the R462/463A mutant; indeed, the mutant exhibited lipid droplet localization instead (Figure 5B). Thus, Arg462 and Arg463 residues are important for the plasma membrane localization of ALDH3B3.

The Arg462 residue of ALDH3B3 is not conserved in ALDH3B2, which has a Trp residue (Trp462) instead (Figure 5A). The involvement of Trp residues in intracellular localization has been reported for a few lipid droplet proteins [32-34]. ALDH3B2 contains another Trp residue (Trp469), which is conserved in ALDH3B3, and which is located between Trp462 and the prenylation site (Figure 5A). We created mutants of these Trp residues (single Ala-substituted mutants $\mathrm{W} 462 \mathrm{~A}$ and $\mathrm{W} 469 \mathrm{~A}$ and a double Ala-substituted mutant W462/469A) and examined their intracellular localization. The lipid droplet localization observed for wild type ALDH3B2 was greatly affected by the W462A mutation (Figure 6A). ALDH3B2 W462A was localized mainly to the cytosol, although some lipid droplet localization was retained. However, ALDH3B2 W469A and W462/469A mutant proteins were almost completely absent from the lipid droplets (Figure 6A). Consistent with these results, cell fractionation assays revealed that the membrane localization of ALDH3B2 W462A was reduced and little ALDH3B2 W469A and W462/469A were detected in the membrane 
fractions. Taken together, these results indicate that the Trp462 and Trp469 residues are important for the lipid droplet localization of ALDH3B2.

We next examined the role of prenylation in the lipid droplet localization of ALDH3B2. We created a Ser-substituted mutant of the prenylated Cys residue (C476S) and subjected cells to analysis by immunofluorescence microscopy. The ALDH3B2 C476S mutant was dispersed throughout the cytosol (Figure 6A), indicating that prenylation is required for lipid droplet localization. This result is consistent with the findings from the cell fractionation experiment with the geranylgeranyltransferase inhibitor, where inhibition of prenylation caused a decrease in membrane-associated ALDH3B2 (Figure 3A). 


\section{DISCUSSION}

The accumulation of aldehydes is cytotoxic because of their high reactivity. ALDHs are responsible for the detoxification of aldehyde molecules by oxidizing them to carboxylic acids [1]. Lipids are one source of cellular aldehydes; these lipid-derived aldehydes are generated via the normal metabolism of several lipids as well as the oxidation of unsaturated fatty acids due to oxidative stress. For example, in the metabolic pathway of the lipid mediator S1P, S1P is cleaved to the fatty aldehyde hexadecenal and phosphoethanolamine [14]. Hexadecenal is oxidized to hexadecenoic acid by ALDH3A2 and is further metabolized to glycerophospholipids [13]. ALDH3A2 is the causative gene of the neurocutaneous disorder Sjögren-Larsson syndrome [2], whose pathology is believed to be caused by the accumulation of lipid-derived aldehydes, including hexadecenal [12-14]. Oxidative stress generates free radicals that react with unsaturated fatty acids, and the resulting lipid hydroperoxides are degraded into several compounds, such as 4-HNE from n-6 polyunsaturated fatty acids [35].

Of the 21 mouse ALDHs, it is the ALDH3 subfamily members (ALDH3A1, ALDH3A2, ALDH3B1, ALDH3B2, and ALDH3B3) that are responsible for the oxidation of lipid-derived aldehydes. Although ALDH3A1, ALDH3A2, and ALDH3B1 have already been biochemically analyzed $[9,17,25]$, little research has been conducted on ALDH3B2 or ALDH3B3. In the present study, we characterized both ALDH3B2 and ALDH3B3 with respect to their enzyme activity, intracellular localization, tissue distribution, and lipid modification, and compared them with the other ALDH3 subfamily members. All ALDH3 subfamily members except for ALDH3A1 exhibited activity toward a broad range of medium- and long-chain aliphatic aldehydes, whereas the activity of ALDH3A1 toward the long-chain aldehyde hexadecanal was low (Figure 1A). The substrate preferences of ALDH3 subfamily members corresponded with their membrane localizations. ALDH3A1 was found to be a soluble protein, while the other ALDH3 subfamily members were membrane proteins (Figure 2A). Hexadecanal is more hydrophobic than octanal and is likely to associate with the membrane, where ALDH3A2, ALDH3B1, ALDH3B2, and ALDH3B3 are located. When the prenylation of ALDH3B proteins were inhibited by GGTI-2133, their activity toward hexadecanal decreased (Figure 3B). We have previously obtained similar results with human ALDH3B1 prenylation mutants, which exhibited reduced activity toward hexadecanal compared to wild type ALDH3B1, despite normal activity levels toward octanal [17]. As in this present study, these previous in vitro enzyme assays were performed under non-ionic detergent, Triton X-100-solubilized conditions. Therefore, these results suggest that prenylation does not affect the enzyme activity of ALDH3B1; rather, the micelle association of ALDH3B1 through prenylation may be required for the efficient recognition of hexadecanal. Similar effects may be responsible for the decrease in the enzyme activity of ALDH3 proteins toward hexadecanal upon treatment with GGTI-2133 in this study.

Our results indicate that ALDH3A2, ALDH3B1, ALDH3B2, and ALDH3B3 exhibit similar, broad substrate preferences toward medium- and long-chain aldehydes. Thus, the differences in their functions cannot be attributed to their substrate specificity. However, their intracellular localizations varied greatly; ALDH3A2 is found in the ER and peroxisomes, 
ALDH3B1 and ALDH3B3 are localized to the plasma membrane, and ALDH3B2 locates to lipid droplets [11, 17] (Figure 2B). It therefore follows that they function to remove lipid-derived aldehydes generated at their specific sites of localization. For example, the dominant splicing isoform of ALDH3A2 is localized to the ER, which is a major site of lipid metabolism. S1P metabolism also occurs in the ER and the ER-resident ALDH3A2 oxidizes the S1P metabolite hexadecenal [13].

ALDH3B1 and ALDH3B3 are localized to the plasma membrane. Since the plasma membrane is in contact with the extracellular environment, it is susceptible to exogenous oxidative stress. ALDH3B1 and ALDH3B3 may be responsible for removal of such oxidative stress-induced aldehydes in the plasma membrane. Consistent with this notion, ALDH3B1 protected cells from the cytotoxic, exogenously added aldehydes and oxidants [16]. ALDH3B3 is rodent-specific and is not conserved in humans. It is possible that ALDH3B1 alone is sufficient to remove plasma membrane aldehydes in humans. We found that ALDH3B2 was localized to lipid droplets (Figure 2C and 2D). Triglycerides stored in lipid droplets contain a high amount of unsaturated fatty acids [21], which can be converted to aldehydes under conditions of oxidative stress. ALDH3B2 may be involved in removal of these aldehydes generated in lipid droplets. Lipid droplets are abundant in adipose tissues, consistent with our data demonstrating that the expression level of $A L D H 3 B 2$ mRNA in white adipose tissue is high (Figure 1B).

All ALDH3B proteins (ALDH3B1, ALDH3B2, and ALDH3B3) are modified by prenylation (geranylgeranylation), and inhibition of this modification caused shifts in the localization from the membrane to the soluble fraction (Figure 3A). Previous studies on Ras, another prenylated protein, revealed that prenylation alone is not sufficient for stable plasma membrane association, and that additional palmitoylation (in the cases of N-Ras, H-Ras, and $\mathrm{K}$-RasA) or positively charged residues (in the case of $\mathrm{K}-\mathrm{RasB}$ ) is required [30]. In the plasma membrane, negatively charged phospholipids such as phosphoinositides, phosphatidylinositol, and phosphatidylserine are asymmetrically enriched in the inner leaflets [36] and interact with positively charged residues [31]. We demonstrated here that ALDH3B1 is palmitoylated at Cys462 and Cys463 (Figure 3D) and that the positively charged residues Arg462 and Arg463 are important for the plasma membrane localization of ALDH3B3 (Figure 5B).

We also found that ALDH3B2 was localized to lipid droplets (Figure 2C and 2D). However, mutations in the Trp462 and/or Trp469 as well as Cys476 residues of ALDH3B2 prevent this movement (Figure 6A), demonstrating that both prenylation and the Trp residues are required for lipid droplet localization of ALDH3B2. Trp residues are often present in membrane proteins at the interface between the aqueous phase and the lipids, and are involved in their binding to membranes $[37,38]$. Biophysical analysis using NMR demonstrated that the aromaticity of Trp residues results in a tendency for these residues to reside in the electrostatically complex interface environment while the flat rigid shape of the Trp residue limits access to the hydrophobic moiety of the lipid chain [39]. Although the targeting mechanism of proteins to lipid droplets is largely unclear, the importance of Trp residues for lipid droplet localization has been previously reported for proteins including 
CGI-58, cholesteryl ester transfer protein, and the guanine nucleotide exchange factor GBF1 [32-34]. When the C-terminal region of ALDH3B2 was subjected to helical wheel plotting, the Trp462 and Trp469 residues and the prenylated Cys residue (Cys476) were closely oriented (Figure 6C). Thus, it is likely that these two Trp residues are located at the interface of the lipid droplet membrane and the cytosol and bind to lipid droplet membranes. When the positively charged Arg462 and Arg463 residues of ALDH3B3 were substituted with Ala, the mutant protein localized to the lipid droplets (Figure 5B). Since ALDH3B3 contains Trp469, which is conserved in ALDH3B2, the ALDH3B3 R462/463 A mutant protein may be localized to lipid droplets through Trp469.

Lipid droplets have recently garnered much attention, particularly in the last decade, as abnormal quantity of lipid droplets has been linked to several metabolic diseases [18, 19]. However, little is known about the molecular mechanisms underlying protein targeting to lipid droplets or the removal of oxidized lipids from lipid droplets. Future studies further elaborating upon our findings and those of others will be important to elucidate such mechanisms. 


\section{AUTHOR CONTRIBUTION}

Takuya Kitamura designed, performed experiments, and analyzed the data. Shuyu Takagi performed experiments. Tatsuro Naganuma provided scientific advice. Akio Kihara planned the project and wrote the manuscript.

\section{ACKNOWLEDGEMENTS}

We are grateful to Dr. T. Sassa for engaging in useful discussions with us.

\section{FUNDING}

This work was supported by Grant-in-Aids for Scientific Research on Innovative Areas (25116701 and 25117701) to AK from the Japan Society for the Promotion of Science (JSPS). 


\section{REFERENCES}

1 Marchitti, S. A., Brocker, C., Stagos, D. and Vasiliou, V. (2008) Non-P450 aldehyde oxidizing enzymes: the aldehyde dehydrogenase superfamily. Expert Opin. Drug. Metab. Toxicol. 4, 697-720

2 Rizzo, W. B. (2007) Sjögren-Larsson syndrome: molecular genetics and biochemical pathogenesis of fatty aldehyde dehydrogenase deficiency. Mol. Genet. Metab. 90, 1-9

3 Geraghty, M. T., Vaughn, D., Nicholson, A. J., Lin, W. W., Jimenez-Sanchez, G., Obie, C., Flynn, M. P., Valle, D. and Hu, C. A. (1998) Mutations in the $\Delta^{1}$-pyrroline 5-carboxylate dehydrogenase gene cause type II hyperprolinemia. Hum. Mol. Genet. 7, 1411-1415

4 Mills, P. B., Struys, E., Jakobs, C., Plecko, B., Baxter, P., Baumgartner, M., Willemsen, M. A., Omran, H., Tacke, U., Uhlenberg, B., Weschke, B. and Clayton, P. T. (2006) Mutations in antiquitin in individuals with pyridoxine-dependent seizures. Nat. Med. 12, 307-309

5 Akaboshi, S., Hogema, B. M., Novelletto, A., Malaspina, P., Salomons, G. S., Maropoulos, G. D., Jakobs, C., Grompe, M. and Gibson, K. M. (2003) Mutational spectrum of the succinate semialdehyde dehydrogenase (ALDH5A1) gene and functional analysis of 27 novel disease-causing mutations in patients with SSADH deficiency. Hum. Mutat. 22, 442-450

6 Sass, J. O., Walter, M., Shield, J. P., Atherton, A. M., Garg, U., Scott, D., Woods, C. G. and Smith, L. D. (2012) 3-Hydroxyisobutyrate aciduria and mutations in the ALDH6A1 gene coding for methylmalonate semialdehyde dehydrogenase. J. Inherit. Metab. Dis. 35, 437-442

7 Estey, T., Piatigorsky, J., Lassen, N. and Vasiliou, V. (2007) ALDH3A1: a corneal crystallin with diverse functions. Exp. Eye Res. 84, 3-12

8 Lassen, N., Bateman, J. B., Estey, T., Kuszak, J. R., Nees, D. W., Piatigorsky, J., Duester, G., Day, B. J., Huang, J., Hines, L. M. and Vasiliou, V. (2007) Multiple and additive functions of ALDH3A1 and ALDH1A1: cataract phenotype and ocular oxidative damage in Aldh3a1(-/-)/Aldh1a1(-/-) knock-out mice. J. Biol. Chem. 282, 25668-25676

9 Kelson, T. L., Secor McVoy, J. R. and Rizzo, W. B. (1997) Human liver fatty aldehyde dehydrogenase: microsomal localization, purification, and biochemical characterization. Biochim. Biophys. Acta. 1335, 99-110

10 Masaki, R., Yamamoto, A. and Tashiro, Y. (1996) Membrane topology and retention of microsomal aldehyde dehydrogenase in the endoplasmic reticulum. J. Biol. Chem. 271, 16939-16944

11 Ashibe, B., Hirai, T., Higashi, K., Sekimizu, K. and Motojima, K. (2007) Dual subcellular localization in the endoplasmic reticulum and peroxisomes and a vital role in protecting against oxidative stress of fatty aldehyde dehydrogenase are achieved by alternative splicing. J. Biol. Chem. 282, 20763-20773

12 Rizzo, W. B. (2011) The role of fatty aldehyde dehydrogenase in epidermal structure and function. Dermatoendocrinol. 3, 91-99 
13 Nakahara, K., Ohkuni, A., Kitamura, T., Abe, K., Naganuma, T., Ohno, Y., Zoeller, R. A. and Kihara, A. (2012) The Sjögren-Larsson syndrome gene encodes a hexadecenal dehydrogenase of the sphingosine 1-phosphate degradation pathway. Mol. Cell. 46, 461-471

14 Kihara, A. (2014) Sphingosine 1-phosphate is a key metabolite linking sphingolipids to glycerophospholipids. Biochim. Biophys. Acta. 1841, 766-772

15 Marchitti, S. A., Orlicky, D. J. and Vasiliou, V. (2007) Expression and initial characterization of human ALDH3B1. Biochem. Biophys. Res. Commun. 356, 792-798

16 Marchitti, S. A., Brocker, C., Orlicky, D. J. and Vasiliou, V. (2010) Molecular characterization, expression analysis, and role of ALDH3B1 in the cellular protection against oxidative stress. Free Radic. Biol. Med. 49, 1432-1443

17 Kitamura, T., Naganuma, T., Abe, K., Nakahara, K., Ohno, Y. and Kihara, A. (2013) Substrate specificity, plasma membrane localization, and lipid modification of the aldehyde dehydrogenase ALDH3B1. Biochim. Biophys. Acta. 1831, 1395-1401

18 Krahmer, N., Farese, R. V., Jr. and Walther, T. C. (2013) Balancing the fat: lipid droplets and human disease. EMBO Mol. Med. 5, 905-915

19 Sahini, N. and Borlak, J. (2014) Recent insights into the molecular pathophysiology of lipid droplet formation in hepatocytes. Prog. Lipid Res. 54, 86-112

20 Penno, A., Hackenbroich, G. and Thiele, C. (2013) Phospholipids and lipid droplets. Biochim. Biophys. Acta. 1831, 589-594

21 Hodson, L., Skeaff, C. M. and Fielding, B. A. (2008) Fatty acid composition of adipose tissue and blood in humans and its use as a biomarker of dietary intake. Prog. Lipid Res. 47, 348-380

22 Ikeda, M., Kanao, Y., Yamanaka, M., Sakuraba, H., Mizutani, Y., Igarashi, Y. and Kihara, A. (2008) Characterization of four mammalian 3-hydroxyacyl-CoA dehydratases involved in very long-chain fatty acid synthesis. FEBS Lett. 582, 2435-2440

23 Kariya, Y., Kihara, A., Ikeda, M., Kikuchi, F., Nakamura, S., Hashimoto, S., Choi, C. H., Lee, Y. M. and Igarashi, Y. (2005) Products by the sphingosine kinase/sphingosine 1-phosphate (S1P) lyase pathway but not S1P stimulate mitogenesis. Genes Cells. 10, 605-615

24 Ohno, Y., Kashio, A., Ogata, R., Ishitomi, A., Yamazaki, Y. and Kihara, A. (2012) Analysis of substrate specificity of human DHHC protein acyltransferases using a yeast expression system. Mol. Biol. Cell. 23, 4543-4551

25 Pappa, A., Estey, T., Manzer, R., Brown, D. and Vasiliou, V. (2003) Human aldehyde dehydrogenase 3A1 (ALDH3A1): biochemical characterization and immunohistochemical localization in the cornea. Biochem. J. 376, 615-623

26 Nees, D. W., Wawrousek, E. F., Robison, W. G., Jr. and Piatigorsky, J. (2002) Structurally normal corneas in aldehyde dehydrogenase 3a1-deficient mice. Mol. Cell. Biol. 22, 849-855

27 Berndt, N. and Sebti, S. M. (2011) Measurement of protein farnesylation and geranylgeranylation in vitro, in cultured cells and in biopsies, and the effects of prenyl 
transferase inhibitors. Nat. Protoc. 6, 1775-1791

28 Resh, M. D. (1999) Fatty acylation of proteins: new insights into membrane targeting of myristoylated and palmitoylated proteins. Biochim. Biophys. Acta. 1451, 1-16

29 Wan, J., Roth, A. F., Bailey, A. O. and Davis, N. G. (2007) Palmitoylated proteins: purification and identification. Nat. Protoc. 2, 1573-1584

30 Hancock, J. F., Paterson, H. and Marshall, C. J. (1990) A polybasic domain or palmitoylation is required in addition to the CAAX motif to localize $\mathrm{p} 21^{\text {ras }}$ to the plasma membrane. Cell. 63, 133-139

31 Johnson, J. L., Erickson, J. W. and Cerione, R. A. (2012) C-terminal di-arginine motif of $\mathrm{Cdc} 42$ protein is essential for binding to phosphatidylinositol 4,5-bisphosphate-containing membranes and inducing cellular transformation. J. Biol. Chem. 287, 5764-5774

32 Gruber, A., Cornaciu, I., Lass, A., Schweiger, M., Poeschl, M., Eder, C., Kumari, M., Schoiswohl, G., Wolinski, H., Kohlwein, S. D., Zechner, R., Zimmermann, R. and Oberer, M. (2010) The N-terminal region of comparative gene identification-58 (CGI-58) is important for lipid droplet binding and activation of adipose triglyceride lipase. J. Biol. Chem. 285, 12289-12298

33 Bouvet, S., Golinelli-Cohen, M. P., Contremoulins, V. and Jackson, C. L. (2013) Targeting of the Arf-GEF GBF1 to lipid droplets and Golgi membranes. J. Cell Sci. 126, 4794-4805

34 Koivuniemi, A., Vuorela, T., Kovanen, P. T., Vattulainen, I. and Hyvonen, M. T. (2012) Lipid exchange mechanism of the cholesteryl ester transfer protein clarified by atomistic and coarse-grained simulations. PLoS Comput. Biol. 8, e1002299

35 Cátala, A. (2009) Lipid peroxidation of membrane phospholipids generates hydroxy-alkenals and oxidized phospholipids active in physiological and/or pathological conditions. Chem. Phys. Lipids. 157, 1-11

36 Ikeda, M., Kihara, A. and Igarashi, Y. (2006) Lipid asymmetry of the eukaryotic plasma membrane: functions and related enzymes. Biol. Pharm. Bull. 29, 1542-1546

37 Landolt-Marticorena, C., Williams, K. A., Deber, C. M. and Reithmeier, R. A. (1993) Non-random distribution of amino acids in the transmembrane segments of human type I single span membrane proteins. J. Mol. Biol. 229, 602-608

38 Gelb, M. H., Cho, W. and Wilton, D. C. (1999) Interfacial binding of secreted phospholipases $\mathrm{A}_{2}$ : more than electrostatics and a major role for tryptophan. Curr. Opin. Struct. Biol. 9, 428-432

39 Yau, W. M., Wimley, W. C., Gawrisch, K. and White, S. H. (1998) The preference of tryptophan for membrane interfaces. Biochemistry. 37, 14713-14718 


\section{FIGURE LEGENDS}

\section{FIGURE 1. Substrate specificity and tissue distribution of ALDH3B2 and ALDH3B3.}

(A) HEK $293 \mathrm{~T}$ cells were transfected with the pCE-puro 3xFLAG-mALDH3A1, pCE-puro 3xFLAG-mALDH3A2, pCE-puro 3xFLAG-mALDH3B1, pCE-puro 3xFLAG-mALDH3B2, or pCE-puro 3xFLAG-mALDH3B3 plasmid. Twenty-four hours after transfection, the total lysate was prepared from these cells, solubilized with Triton X-100, and subjected to affinity-purification using anti-FLAG M2 beads. The affinity-purified protein (10 ng) was incubated with $500 \mu \mathrm{M} \mathrm{NAD}^{+}$and $100 \mu \mathrm{M}$ hexadecanal, octanal, or acetaldehyde for $15 \mathrm{~min}$ at $37{ }^{\circ} \mathrm{C}$. The fluorescence of the NADH product was measured using a monochromator Infinite M200. Values represent the mean \pm S.D. of three independent experiments. (B) The total RNA prepared from the liver, kidney, lung, testis, small intestine, white adipose tissue (WAT), and spleen of 4-8 week-old C57BL/6 mice was subjected to real-time quantitative RT-PCR using primers specific to $A L D H 3 B 2, A L D H 3 B 3$, and GAPDH. Values presented are the amount of $A L D H 3 B$ mRNA from each tissue type relative to that of $G A P D H$, and represent the means \pm S.D. from three independent reactions.

\section{FIGURE 2. Intracellular localization sites differ among ALDH3 proteins.}

(A) HeLa cells were transfected with the pCE-puro 3xFLAG-mALDH3A1, pCE-puro 3xFLAG-mALDH3A2, pCE-puro 3xFLAG-mALDH3B1, pCE-puro 3xFLAG-mALDH3B2, or pCE-puro 3xFLAG-mALDH3B3 plasmid. Twenty-four hours after transfection, the total lysate was prepared from these cells and centrifuged at $100,000 \mathrm{~g}$ for $30 \mathrm{~min}$ at $4{ }^{\circ} \mathrm{C}$. The resulting supernatant (soluble fraction; $\mathrm{S}$ ) and pellet (membrane fraction; $\mathrm{M}$ ) were subjected to immunoblotting using anti-FLAG, anti-calnexin (a membrane protein marker), and anti-GAPDH (a soluble protein marker) antibodies. (B) HeLa cells were transfected with the pEGFP-C1 (vector), pEGFP-mALDH3B1, pEGFP-mALDH3B2, or pEGFP-mALDH3B3 plasmid. Forty-eight hours after transfection, cells were observed microscopically with a Leica DM5000B fluorescence microscope. (C and D) HeLa cells were transfected with the pCE-puro 3xFLAG-mALDH3B2 plasmid (C) or pEGFP-mALDH3B2 and pCE-puro 3xFLAG-CGI-58 plasmids (D). Twenty-four hours after transfection, cells were treated with $100 \mu \mathrm{M}$ oleic acid and incubated for a further $24 \mathrm{~h}$. Cells were then fixed with $3.7 \%$ formaldehyde, stained with oil red $O(\mathbf{C})$ and/or anti-FLAG antibody (C and D, respectively), and observed with a Leica DM5000B fluorescence microscope. Bar: $25 \mu \mathrm{m}$.

FIGURE 3. Geranylgeranylation of ALDH3B proteins and its importance in enzymatic activity. (A and B) HeLa cells were transfected with the pCE-puro 3xFLAG-mALDH3B1, pCE-puro 3xFLAG-mALDH3B2, or pCE-puro 3xFLAG-mALDH3B3 plasmid. Three hours after transfection, cells were incubated with $10 \mu \mathrm{M}$ GGTI-2133, a geranylgeranyltransferase I inhibitor, or its DMSO solvent for $24 \mathrm{~h}$ at $37{ }^{\circ} \mathrm{C}$. (A) The total cell lysate was prepared and centrifuged at $100,000 \mathrm{~g}$ for $30 \mathrm{~min}$ at $4{ }^{\circ} \mathrm{C}$. The resulting supernatant (soluble fraction; S) and pellet (membrane fraction; $\mathrm{M}$ ) were then subjected to immunoblotting using anti-FLAG, anti-calnexin (a membrane protein marker), and anti-GAPDH (a soluble protein marker) antibodies. (B) The total lysate was prepared, solubilized with Triton X-100, and subjected to 
affinity-purification using anti-FLAG M2 beads. The affinity-purified protein (10 ng) was incubated with $500 \mu \mathrm{M} \mathrm{NAD}^{+}$and $100 \mu \mathrm{M}$ hexadecanal for $15 \mathrm{~min}$ at $37^{\circ} \mathrm{C}$. The fluorescence of the NADH product was measured using a monochromator Infinite M200. Values represent the mean \pm S.D. of three independent experiments. Statistically significant differences are indicated $\left({ }^{*} p<0.01,{ }^{*} p<0.05 ; t\right.$-test). (C and D), HEK 293T cells were transfected with the pCE-puro 3xFLAG-mALDH3B1 (C and D), pCE-puro 3xFLAG-mALDH3B2 (C), pCE-puro 3xFLAG-mALDH3B3 (C), pCE-puro 3xFLAG-mALDH3B1-C462S (D), pCE-puro 3xFLAG-mALDH3B1-C463S (D), or pCE-puro 3xFLAG-mALDH3B1-C462/463S (D) plasmid. The total cell lysate was prepared, treated with $N$-ethylmaleimide to block non-palmitoylated Cys residues, and incubated with hydroxylamine (Ha) to liberate palmitic acid. The exposed Cys residues were biotinylated using EZ-LinkTM Biotin-HPDP (Thermo Fisher Scientific). The biotinylated proteins were precipitated with immobilized avidin beads and detected by immunoblotting with anti-FLAG antibody (upper panels). The ALDH3B proteins in the total lysate were detected by immunoblotting with anti-FLAG antibody (lower panels). AvP, avidin precipitation; IB, immunoblotting.

FIGURE 4. The C-terminal regions of ALDH3B2 and ALDH3B3 are important for their localization.

HeLa cells were transfected with the pEGFP-mALDH3B2, pEGFP-mALDH3B3, pEGFP-mALDH3B(3/2/2), pEGFP-mALDH3B(2/3/2), pEGFP-mALDH3B(2/2/3), pEGFP-mALDH3B(2/3/3), pEGFP-mALDH3B(3/2/3), or pEGFP-mALDH3B(3/3/2) plasmid. Each plasmid encodes for ALDH3B2, ALDH3B3, or the ALDH3B2/ALDH3B3 chimera protein as illustrated. Forty-eight hours after transfection, cells were subjected to microscopic observation with a Leica DM5000B fluorescence microscope.

FIGURE 5. The Arg462 and Arg463 residues are important for the plasma membrane localization of ALDH3B3. (A) The C-terminal sequences of ALDH3B1, ALDH3B2, and ALDH3B3 are presented. The C-terminal prenylation motifs are highlighted in pink. The Arg, Trp, and palmitoylated Cys residues are shown as blue, orange, and red, respectively. The Arg residues mutated in this study are underlined. (B) HeLa cells were transfected with the pEGFP-mALDH3B3 or pEGFP-mALDH3B3-R462/463A plasmid. Twenty-four hours after transfection, cells were treated with $100 \mu \mathrm{M}$ oleic acid and incubated for a further $24 \mathrm{~h}$. Cells were fixed with $3.7 \%$ formaldehyde, stained with oil red $O$, and observed under a Leica DM5000B fluorescence microscope. Bar, $25 \mu \mathrm{m}$.

FIGURE 6. The residues Trp462 and Trp469 are important for the lipid droplet localization of ALDH3B2.

(A and B) HeLa cells were transfected with the pCE-puro 3xFLAG-mALDH3B2, pCE-puro 3xFLAG-mALDH3B2-W462A, pCE-puro 3xFLAG-mALDH3B2-W469A, pCE-puro 3xFLAG-mALDH3B2-W462/469A, or pCE-puro 3xFLAG-mALDH3B2-C476S plasmid. (A) Twenty-four hours after transfection, cells were treated with $100 \mu \mathrm{M}$ oleic acid and incubated for a further $24 \mathrm{~h}$. Cells were fixed with $3.7 \%$ formaldehyde, stained with anti-FLAG 
antibody and oil red $O$, and observed under a Leica DM5000B fluorescence microscope. Bar, $25 \mu \mathrm{m}$. (B) Twenty-four hours after transfection, total cell lysates were prepared and separated into the soluble fraction (S) and the membrane fraction (M) by centrifugation at $100,000 \mathrm{~g}$ for $30 \mathrm{~min}$ at $4{ }^{\circ} \mathrm{C}$. Proteins were then subjected to immunoblotting using anti-FLAG, anti-calnexin (a membrane protein marker), and anti-GAPDH (a soluble protein marker) antibodies. (C) The C-terminal sequence of ALDH3B2 was subjected to helical wheel plotting using Heliquest software (heliquest.ipmc.cnrs.fr/). The Cys residue shown in the red circle represents the prenylated residue. 
Table 1. Nucleotide sequences of primers used in this study

\begin{tabular}{|c|c|}
\hline Primer & Nucleotide sequence \\
\hline ALDH3A1-F1 & 5'-GGATCCATGAGCAATATCAGTAGCATCGTGA-3' (BamHI) \\
\hline ALDH3A1-R1 & 5'-TCAATGCCGGGGCATCTTGGCTGGG-3' \\
\hline ALDH3A2-F1 & 5'-GGATCCATGGAGCGCCAGGTCCTACGGCTTC-3' (BamHI) \\
\hline ALDH3A2-R1 & 5'-TTACAGCTGATCCTTGACAATCACA-3' \\
\hline ALDH3A1-F1 & 5'-GGATCCATGGACTCGTTTGAAGACAAGCTTC-3' (BamHI) \\
\hline ALDH3B1-R1 & 5'-TTACAGCAGGGTGCAGCTGCAGCAC-3' \\
\hline ALDH3B2-F1 & 5'-GGATCCATGTCTGCTGCAGAGACAGGCTCGG-3' (BamHI) \\
\hline ALDH3B2-R1 & 5'-TCACAGGAGGGTGCAGCTCCGGGAG-3' \\
\hline ALDH3B2-R2 & 5'-TCACAGGAGGGTGGAGCTCCGGGAGCCTATGGCCCAGCT-3' \\
\hline ALDH3B3-F1 & 5'-GGATCCATGTCCACCAAAGGCAAACACCCAC-3' (BamHI) \\
\hline ALDH3B3-R1 & 5'-TTACAGAAGGGTGCAGCTCTCAGAG-3' \\
\hline CGI-58-F1 & 5'-GGATCCATGGCGGCGGAGGAGGAGGAGGTGG-3' (BamHI) \\
\hline CGI-58-R1 & 5'-TCAGTCCACAGTGTCGCAGATCTCC-3' \\
\hline ALDH3B2-Q1F & 5'-CCGCCTTATGGTCCCGCGAACCAACAACTGAT-3' \\
\hline ALDH3B2-Q1R & 5'-ATCAGTTGTTGGTTCGCGGGACCATAAGGCGG-3' \\
\hline ALDH3B2-Q2F & 5'-CAACAACTGATAAGCGCGGCCATAGGCTCCCG-3' \\
\hline ALDH3B2-Q2R & 5'-CGGGAGCCTATGGCCGCGCTTATCAGTTGTTG-3' \\
\hline ALDH3B3-Q1F & 5'-CCGCCCTATAGTCCCGCAGCGCAGCAGCTGCTAAGA-3' \\
\hline ALDH3B3-Q1R & 5'-TCTTAGCAGCTGCTGCGCTGCGGGACTATAGGGCGG-3' \\
\hline ALDH3B2-rt1 & 5'-TGAGTTCATCAACCGGCGGGAGAAGC-3' \\
\hline ALDH3B2-rt2 & 5'-GTTGTTGGTTCCAGGGACCATAAGG-3' \\
\hline ALDH3B3-rt1 & 5'-CTTTATGCCTATTCCAACAACGCAG-3' \\
\hline ALDH3B3-rt2 & 5'-GGGTGCAGCTCTCAGAGCCGATAGC-3' \\
\hline GAPDH-rt1 & 5'-GAACGGGAAGCTCACTGGCATGGCC-3' \\
\hline GAPDH-rt2 & 5'-TGTCATACCAGGAAATGAGCTTGAC-3' \\
\hline
\end{tabular}

The restriction sites created are underlined. 
Figure 1

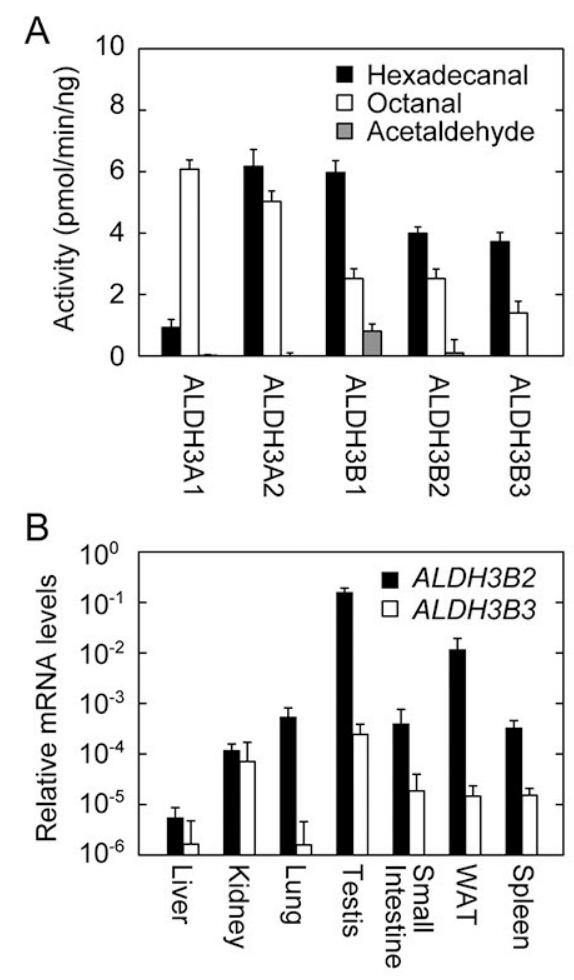


Figure 2

A

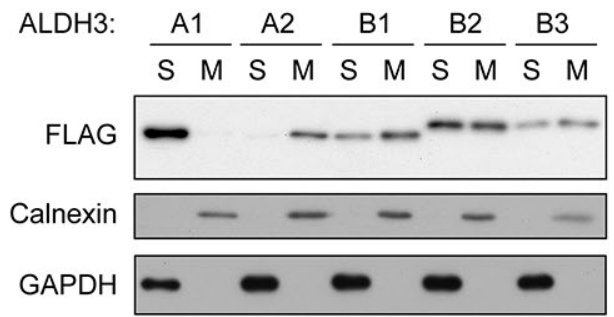

B

EGFP

ALDH3B1
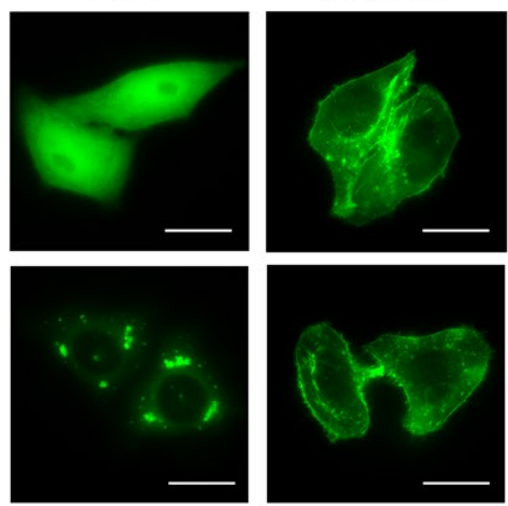

ALDH3B2

ALDH3B3

C

ALDH3B2

Oil red $O$
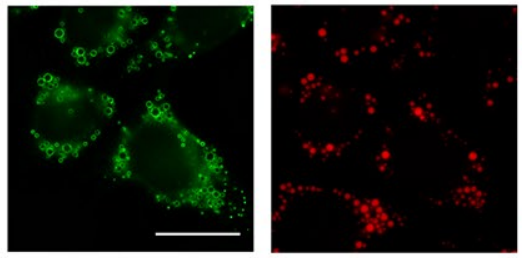

CGI-58

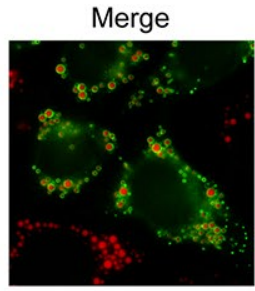

D

ALDH3B2

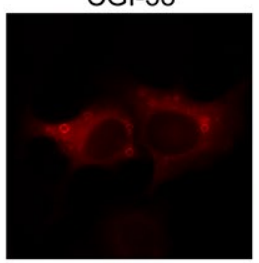

Merge
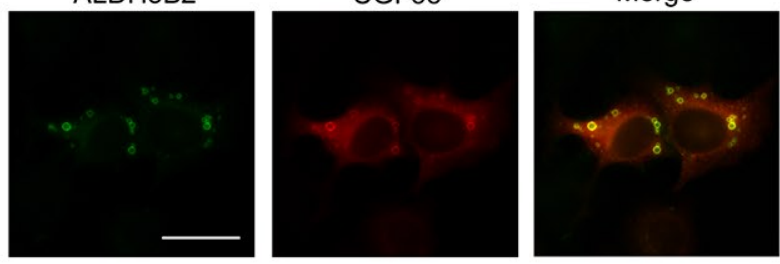
Figure 3

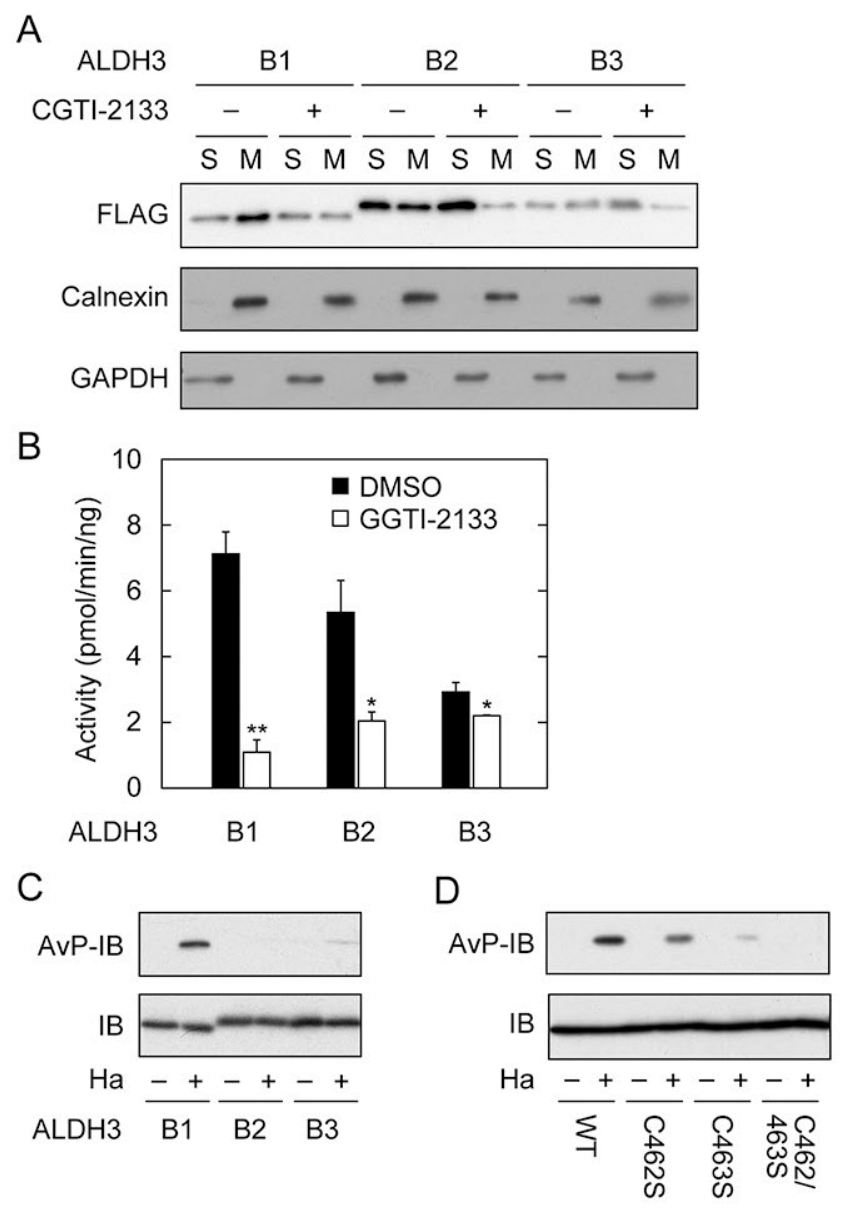


Figure 4

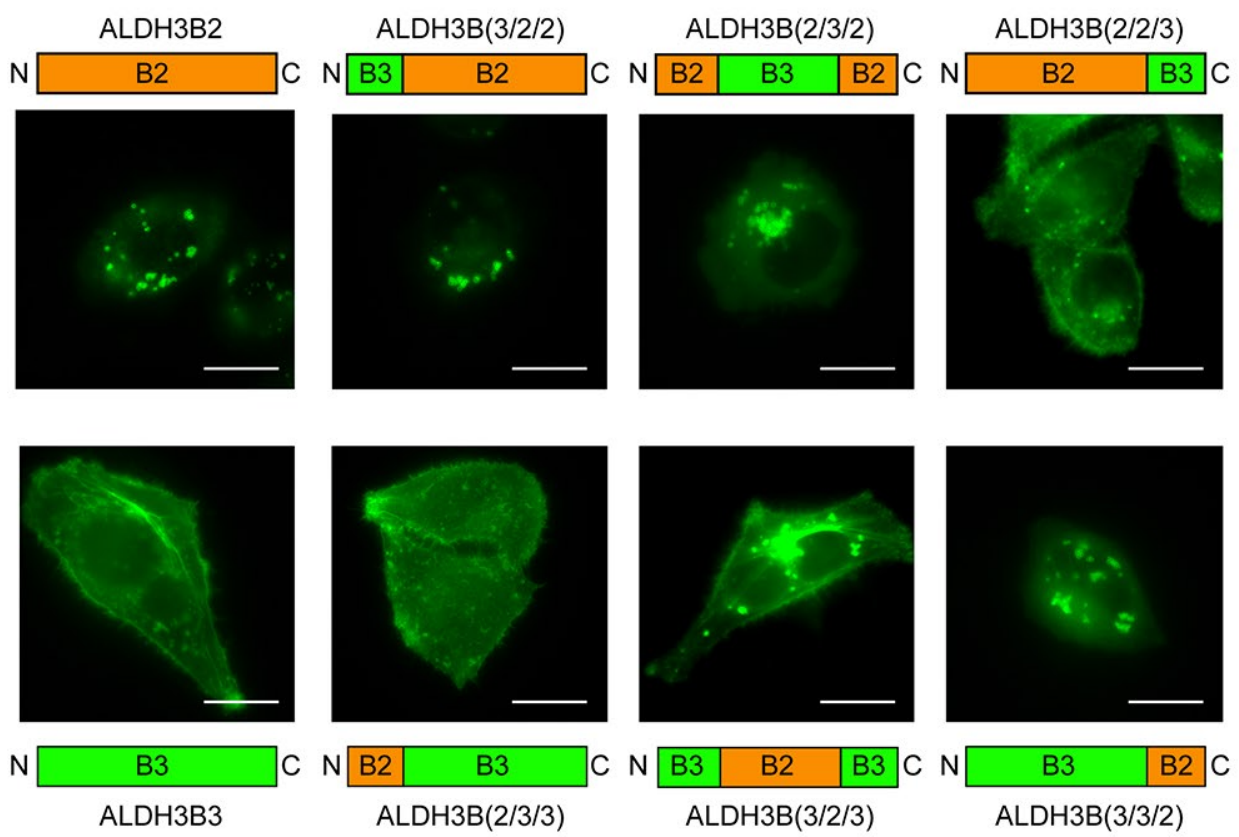


Figure 5

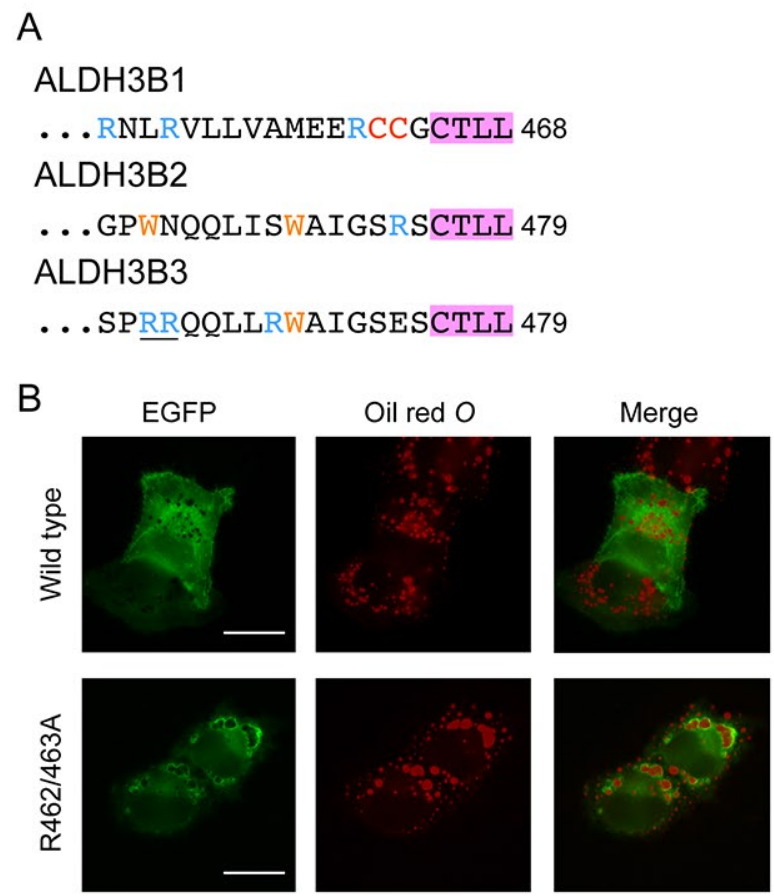


Figure 6

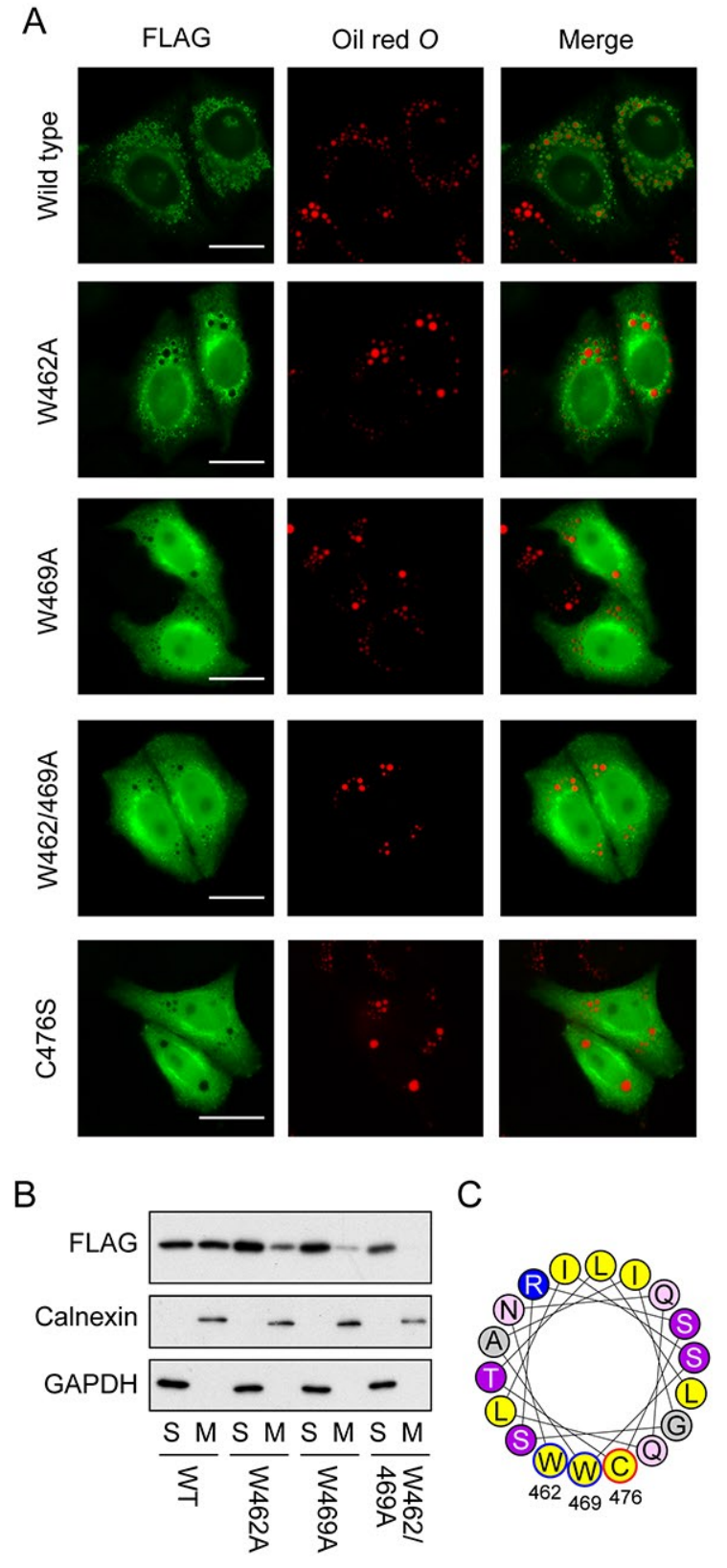

